\title{
LETTER
}

doi:10.1017/S1041610216002404

\section{Elder abuse by abandonment in India: a novel community awareness and intervention strategy}

India, along with the rest of the world, is aging rapidly, but more so. Of a total population of over 1.2 billion, the aging population over 60 years is 109 million, projected to rise above 300 million by the year 2050, exceeding the younger population. One important ramification of this highly populous and under-resourced setting is that it provides a ripe environment for elder abuse (Chokkanathan and Lee, 2005), a problem besetting vulnerable aging populations globally (Patterson and Ploeg, 2007).

By illustration, India's Hindi festival of KumbhMela, where every 12 years, over 10 million people gather to take part in holy bathing days at a range of sites, provides an opportunity for abandonment of elderly disabled people, usually women. Although there is no data documenting the extent of this problem, estimates of abandoned women range from hundreds to thousands, observed in KumbhMela festivals in 1989, 2001, 2010, 2013, and 2015 (Spinney, 2013).

Community responses to this problem have been limited to lost and found camps and public "PA announcements" (Spinney, 2013) prompting the first author Mangal Kardile (MK) to explore strategies with the local police in 2001 in Nasik while relocating and re-uniting the abandoned elder population, and giving shelter for those who could not be re-united. One of the prime issues identified in this "pilot" work was the need for community personnel providing assistance to elders to understand why many could not recall either their names or residential addresses. The association between dementia and elder abuse has been observed across cultures (Patterson and Ploeg, 2007).

In accordance with the World Health Organization's (2008) call for multidisciplinary, interdisciplinary community based approaches to elder abuse, the first author (MK) developed a community education and awareness program on abuse and dementia, tailored and delivered to local police who support the Kumbh-Mela festival. The intervention was delivered from the

Correspondence should be addressed to: Mangal S Kardile, Mental Health Aims, 5/87 Shubhankaroti Bunglow, Vidya Vihar Colony, Nasik 422101, India. Email: kardile.mangal@gmail.com. Phone: +91-985-0645934, +91253-2462132. 7th July 2015 to the 5th August 2015 prior to that year's Kumbh-Mela event and comprised a lecture series and the provision of resource posters. A single lecture "Dementia awareness raising and its significance to Kumbh-Mela" was delivered to 15 police stations. The 30-45 minute lecture included topics relating to understanding normal human brain structures and the relationship with cognitive function, dementia and how this knowledge might help the Police department to handle the lost and/or abandoned population. Stress management techniques and self-care for police were also briefly discussed. A poster written in regional language, displayed in each police station, was designed to assist police in reuniting the disorientated and lost with their families. For example, by using the mistaken place of orientation (i.e. instead of Nasik) as a cue to know the person's own town or at least a place of significance in their life, as well as the person's former (maiden) name if a woman, name of town, and its significant landmarks (e.g., water-well, grocery shop, and bus-stop), or name of siblings, parents and grandparents.

Of the 1433 police staff recipients of education, 588 were administered feedback forms the following day which elicited qualitative feedback regarding (i) the impact of the lecture and poster series; (ii) general observations of elder abuse and abandonment during the $2015 \mathrm{Kumbh}-\mathrm{Mela}$ event; and (iii) future recommendations. Feedback was received from 147 of the 588 staff given feedback forms, yielding a response rate of $25 \%$. Police reported that the posters were widely distributed across large and small sub-police stations ("chowkismall police tin shelter") and in turn, police wrote the information from the posters on smaller papers and kept them on their tables.

Feedback from Police in regards to their general observations of abuse and abandonment during the 2015 Kumbh-Mela event included:

1. when asked to nominate the types of complaints registered in police stations, police staff endorsed the following categories (frequency counts unavailable) - forgetfulness, lost people, lost belongings, innocent people cheated by both the public and some Sadhus (holy men);

2. most of the lost persons were elderly, from specific regions in India such as Bihar and Madhya Pradesh states, and appeared to have a problem of forgetfulness, with only about $15 \%$ of the lost population estimated to be able to identify themselves by name; 
3. practical implementation of strategies was demonstrated by several examples of police successfully using the word cueing described above to reunite lost elders with family members or to connect them with the train to their hometown;

4. Police staff adopted recommendations for crowd control and prohibiting risky behaviors such as prohibiting Sadhus from suddenly throwing silver coins into crowds of millions and causing stampedes; by limiting numbers entering the river at any one time; and using more volunteers.

This is not a systematic evaluation. We report a descriptive account of an innovative, albeit brief, community dementia awareness campaign which achieved significant dissemination amongst community Police staff. Qualitative feedback, albeit impressionistic only, and limited by the response rate, does suggest a value for targeted dementia awareness raising in this context. Although feedback suggested impact on police behavior and support of older people, this clearly needs more methodical investigation with testing of knowledge translation and behavior outcomes.

Moreover, merely returning lost elders, mostly women, to their abandoning families will not address the underlying etiologies of abuse in the Indian social context. These include high levels of economic dependence on children, longstanding vulnerability of widows, illiteracy, and unidentified and untreated physical and neurodegenerative disorders, in a culture transitioning between modernism and traditionalism where modernization and urbanization have weakened traditional family structure (Chokkanathan and Lee, 2005; Das et al., 2012).

The need to promote training in dementia awareness for public contact staff such as Police service personnel to better cater to people living with dementia has long been recognized (Shanley et al., 2004). In the peculiarly public and mass abandonment setting of Kumbh-Mela, Police may be a valuable community resource for identifying and returning lost and abandoned elders. Although in Western contexts most education and prevention training programs for elder abuse have focused on health care professionals, the role of Police in elder abuse strategies, albeit in a far more focused way with individual Police liaison or Vulnerable Community Support officers, has also been explored.

The provision of effective safeguards to prevent abuse is a human right owed to all elders (World Health Organization, 2008) and, in regards to elders with disability such as dementia, an obligation of all 160 signatory countries to the United Nations Convention on the Rights of Persons with Disabilities. How this is done, with limited, or no resources, in a population of 109 million elders is obviously overwhelming. However, educating 1,433 police staff might be a start.

\section{Conflict of interest}

None.

\section{Description of authors' roles}

Mangal Kardile developed and carried out the community awareness project and contributed to the writing of the manuscript. Carmelle Peisah wrote the manuscript.

\section{Acknowledgments}

Mangal Kardile would like to thank Capacity Australia for its support of this and other related research projects.

\section{References}

Chokkanathan, S. and Lee, A. E. (2005). Elder mistreatment in urban India: a community based study. Fournal of Elder Abuse E Neglect, 17, 45-61.

Das, S. K., Pal, S. and Ghosal, M. K. (2012). Dementia: Indian scenario. Neurology India, 60, 618-624.

Patterson, C. and Ploeg, J. (2007). Elder abuse: persistent challenges in recognition and management. Canadian fournal of Geriatrics, 10, 60-63

Shanley, C., Quirke, S., Shaw, L. and Sammut, A. (2004). Working with organizations to implement dementia awareness training for public contact staff. American fournal of Alzheimers Disease and Other Dementias, 19, 166-171.

Spinney, L. (2013). At largest religious festival, some abandon elderly: at the Maha Kumbh Mela in India, families ditch older relatives in the crowds.

http:/news.nationalgeographic.com/news/2013/02/ 130223-culture-travel-religion-india-maha-kumbhmela-world-women-widow-hindu-festival; last accessed 2 January 2017.

World Health Organization. (2008). A global response to elder abuse and neglect: building primary health care capacity to deal with the problem worldwide: main report http://www.who.int/ageing/publications/

ELDER_DocAugust08.pdf; last accessed 2 January 2017.

Mangal S. Kardile ${ }^{1}$

and Carmelle Peisah ${ }^{2,3,4}$

${ }^{1}$ Mental Health Aims, Nasik, India

${ }^{2}$ School of Psychiatry, Faculty of Medicine, University of New South Wales, New South Wales, Australia

${ }^{3}$ Discipline of Psychiatry, Sydney University Medical

School, Sydney, Australia

${ }^{4}$ Capacity Australia, Australia 